\title{
The power of surfaceome analysis in cancer to design novel targeted therapies
}

\author{
Eliana Marinari ${ }^{1,2}$ and Denis Migliorini ${ }^{1,2 *}$ \\ ${ }^{1}$ Department of Oncology and Center for Translational Research in Onco-Hematology, Geneva University Hospitals and University of Geneva, Switzerland \\ ${ }^{2}$ Swiss Cancer Center Leman-SCCL, and Swiss Institute for Experimental Cancer Research - ISREC Foundation, Lausanne, Switzerland
}

The spectrum of cell surface proteins (the surfaceome) is one of the key focus of the drug industry, as $66 \%$ of approved human drugs registered in the Drug Bank database target a protein on the cell-surface, which makes the surfaceome of great therapeutical significance [1]. However, the comprehensive evaluation of the human surface protein repertoire remains a major challenge and several methods are needed to interrogate cell-surface proteins, in particular where low numbers of cells are available.

The combination of gene expression analysis by next generation deep sequencing of bulk RNA-sequencing and single cell RNA-sequencing, integrated with cell-surface protein expression by proteomics, could potentially overcome inaccurate predictions of human cell-surface genes. This approach could avoid relying on an assumed correlation between mRNA levels and effective protein expression and define the cell proteome, including those proteins that are located on the cell surface.

The most advanced NGS-based technologies for transcriptomics are now focused on the analysis of individual cells and allow the characterization of surface markers of rare cell types or subgroups of cells [2]. Moreover, in the past years, proteomic methods have increased their sensitivity, which lead to substantial improvements in mass spectrometry applications [1].

Finally, this approach could constitute a systemic method for target discovery to design novel targeted therapies in cancer and could be particularly useful in the field of immunotherapy and engineered cellular therapies.

Immune cell therapy has made astonishing progress in several cancers, including hematological malignancies and comprises engineered T-cell receptor (TCR), tumor-infiltrating lymphocytes (TILs), cytotoxic T lymphocytes (CTLs), natural killer (NK) cells and chimeric antigen receptor (CAR)-T cells, among others [3]. Importantly, the success of cellular therapy in treating patients is attributable to the antigen choice, which should be ideally a cell surface protein uniformly and strongly expressed in the targeted cancer cells [4]. Other approaches such as multipeptide vaccine provided proof of concept of safety and immunogenicity using cell surface antigens expressed in vivo in malignant glioma [5]. Similarly, the characterization of the full repertoire of tumor antigens may provide more effective immunotherapies with individualized vaccinations, as it has been shown for the Glioma Actively Personalized Vaccine Consortium (GAPVAC) [6].

In addition, one of the major challenges is inter and intra-tumor heterogeneity, as antigens are highly heterogeneous between different patients suffering from the same tumor and not all tumor cells uniformly express the same surface antigens. Together with targeting cancer cells, multi-targeted approaches could be envisaged, by inhibiting cells of the tumor microenvironment, such as pro-tumoral macrophages [7].

\section{Conclusion}

In conclusion, we should not underestimate the importance of the target discovery phase as a critical step to choose a well-selected antigen for novel targeted therapies, to reduce side effects and to maximize anti-tumor efficacy. In this respect, extensive preclinical investigation combining an interdisciplinary approach across bioinformatics and cell biology/cell engineering on the selected antigen is imperative.

\section{References}

1. Bausch-Fluck D, Goldmann U, Muller S (2018) The in silico human surfaceome. Proc Natl Acad Sci USA 115: E10988-E10997.

2. Hwang B, Lee JH, Bang D (2018) Single-cell RNA sequencing technologies and bioinformatics pipelines. Exp Mol Med 50.

3. Rafei H, Mehta RS, Rezvani K (2019) Editorial: Cellular therapies in cancer. Front Immunol 10: 2788.

4. Abbott RC, Cross RS, Jenkins MR (2020) Finding the keys to the CAR: Identifying novel target antigens for $\mathrm{T}$ cell redirection immunotherapies. International Journal of Molecular Sciences 21

5. Migliorini D, Dutoit V, Allard M (2019) Phase I/II trial testing safety and immunogenicity of the multipeptide IMA950/poly-ICLC vaccine in newly diagnosed adult malignant astrocytoma patients. Neuro Oncol 21: 923-933.

6. Hilf N, Kuttruff-Coqui S, Frenzel K (2019) Actively personalized vaccination trial for newly diagnosed glioblastoma. Nature 565: 240-245.

7. Joyce JA (2005) Therapeutic targeting of the tumor microenvironment. Cancer Cell 7: 513-520.
Copyright: (C2020 Marinari E. This is an open-access article distributed under the terms of the Creative Commons Attribution License, which permits unrestricted use, distribution, and reproduction in any medium, provided the original author and source are credited.
${ }^{\star}$ Correspondence to: Denis Migliorini, Professor, Department of Oncology, University Hospital of Geneva, Switzerland, Swiss Cancer Center Léman and ISREC Foundation, Lausanne, Switzerland, E-mail: Denis.Migliorini@hcuge.ch

Received: April 03, 2020; Accepted: April 16, 2020; Published: April 20, 2020 